\title{
PCCP
}

ARTICLE TYPE

Cite this: DOI: $00.0000 / x x x x x x x x x x$

\section{Advancing Predictions of Protein Stability in the Solid State ${ }^{\dagger}$}

\author{
Maarten Batens, ${ }^{b \ddagger}$ Talia A. Shmool, ${ }^{\ddagger}{ }^{\ddagger}$ Jan Massant, ${ }^{d \ddagger}$ J. Axel Zeitler, ${ }^{d \ddagger}$ and \\ Guy Van den Mooter*a
}

Received Date

Accepted Date

DOI: $00.0000 / x x x x x x x x x x$

The $\beta$-relaxation associated with the sub-glass transition temperature $\left(T_{\mathrm{g}, \beta}\right)$ is attributed to fast, localised molecular motions which can occur below the primary glass transition temperature $\left(T_{g \alpha}\right)$. Consistent with $T_{\mathrm{g}, \beta}$ being observed well-below storage temperatures, the $\beta$-relaxation associated motions have been hypothesised to influence protein stability in the solid state and could thus impact the quality of, e.g. protein powders for inhalation or reconstitution and injection.

Why then do distinct solid state protein formulations with similar aggregation profiles after drying and immediate reconstitution, display different profiles when reconstituted following prolonged storage? Is the value of $T_{\mathrm{g}, \beta}$, associated with the beta-relaxation process of the system, a reliable parameter for characterising the behaviour of proteins in the solid state? Bearing this in mind, in this work we further explore the different relaxation dynamics of glassy solid state monoclonal antibody formulations using terahertz time-domain spectroscopy and dynamical mechanical analysis. By conducting a 52-week stability study on a series of multi-component spray-dried formulations, an approach for characterising and analysing the solid-state dynamics and how these relate to protein stability is outlined.

\section{Introduction}

Solid state protein formulations, e.g. protein powders for inhalation or reconstitution and injection, with different compositions but similar aggregation profiles after drying and immediate reconstitution, display different aggregation profiles when reconstituted following prolonged storage. Such formulations are predominantly amorphous and effective stabilisation of proteins requires the protein and excipients to form a single phase without undergoing phase separation during preparation or storage ${ }^{1-3}$. The efficacy of the used excipients has been attributed to their potential to slow down fluctuations in local conformations of the protein, as such changes in conformation are suggested to play a role in protein aggregation upon reconstitution and hence loss of therapeutic function of the protein ${ }^{3,4}$. Even though the primary glass transition or $\alpha$-relaxation temperature is traditionally used

\footnotetext{
* Corresponding author

${ }^{a}$ Drug Delivery and Disposition, KU Leuven, Leuven, Belgium. Tel: +32 163303 04;

E-mail: guy.vandenmooter@kuleuven.be

${ }^{b}$ Drug Delivery and Disposition, KU Leuven, Leuven, Belgium.

${ }^{c}$ Biological Formulation Development, UCB Pharma, Braine l'Alleud, Belgium.

${ }^{d}$ Department of Chemical Engineering and Biotechnology, University of Cambridge, Cambridge, UK.

$\dagger$ Electronic Supplementary Information (ESI) available: [details of any supplementary information available should be included here]. See DOI: 10.1039/cXCP00000x/

$\$$ These authors contributed equally to this work
}

to estimate the (storage) stability of amorphous pharmaceutical solids, it is widely acknowledged that this parameter generally correlates poorly to protein stability in the solid state ${ }^{5}$.

The primary glass transition is certainly an important descriptor for the characterisation of amorphous samples. It is fundamentally linked to the slow-down in large amplitude molecular motions that occur on a relatively slow time-scale. In addition, it is well known that a faster time scale secondary transition, also referred to as $\beta$-relaxation with its own glass transition temperature $\left(T_{\mathrm{g}, \beta}\right)$ exists at temperatures below $T_{\mathrm{g}, \alpha}$. It has been hypothesised that the faster, more localised molecular motions, i.e. those that we referred to as fast $\left(\beta_{\text {fast }}\right)$ as well as Johari-Goldstein relaxations $\left(\beta_{\mathrm{JG}}\right)$, are highly important with regards to protein stability in the solid state ${ }^{5}$. Exactly how these different timescale relaxations relate to each other, i.e. whether or not the faster relaxations are precursors of the $\alpha$-relaxation of a glass, or whether they are separate, overlapping relaxation processes following Arrhenius- $(\beta)$ and non-Arrhenius $(\alpha)$ type temperature dependence, remains a topic for discussion ${ }^{6,7}$. However, it is clear that for the $\beta$-relaxation motions to take place, local energy barriers on the potential energy surface (PES) must be overcome through thermal activation. In this regard it is important to note that, while the PES, i.e. the energy minima, or metabasins present, is not temperature dependent, the exploration of the PES is ${ }^{8}$. Additionally, some authors discern motions within a glass due 
to molecular side chains as non-JG relaxations ${ }^{9}$.

One framework to rationalise the $\beta$-relaxation was developed using the so-called coupling model, with results showing a successful representation of the relaxation times of both the JG- and $\alpha$-relaxations ${ }^{9}$. Based on this model the concept of caged dynamics was introduced to elucidate the motions that occur when the secondary glass transition temperature is reached, which is based upon the molecular structures of viscous liquids. The coupling model asserts that molecules are caged at lower temperatures by the non-harmonic intermolecular potential which dissipates when sufficient thermal energy is supplied at $T_{\mathrm{g}, \beta}{ }^{10,11}$. The cage is formed by a molecule's nearest neighbouring molecules ${ }^{12}$. While the mathematical formalism introduced by Ngai ${ }^{10,11}$ does not depend on a physical picture, post-hoc associations have been made in these publications. However, these do not rely on the ideas put forward by Leporini ${ }^{12}$, and neither model includes assumptions regarding whether only pairwise interactions are significant, or whether the extended interactions could be important. However, because this hypothesis assumes that only intermolecular forces are responsible for the barrier in $\beta$-relaxation, it does not account for intramolecular interactions and complex coupled internal and external motions that are able to take place more freely with increasing temperatures as the free volume increases. It also fails to consider extended intermolecular interactions as opposed to simply these with the nearest neighbours. The interested reader is referred to the work of Dyre ${ }^{13}$ and Simons ${ }^{14}$ on this subject. Aforementioned aspects are expected to be of particular significance in proteins, macromolecules and their formulations, where folding and intramolecular interactions are non-negligible factors in the governing of their energetics. Recent work based on entropic data utilising the principles of JG-relaxation strongly suggest that a glass that has reached $T_{\mathrm{g}, \beta}$ represents rotational or translational motions of a small number of molecules within the bulk sample 15,16 , and not due to cooperative motion throughout the entire bulk of the sample. Additionally, Cicerone and Tyagi have recently shown metabasin transitions, i.e. transitions between energy minima, to be the Johari-Goldstein relaxation events ${ }^{17}$. These principles can be directly translated to macromolecules such as proteins, where individual parts or subunits of the molecule can exhibit substantial motion upon heating and where a distribution of motions can be active within the bulk of the sample, so long as the local potential energy barrier has been overcome.

In previous work, we have shown that terahertz time-domain spectroscopy (THz-TDS) can be used to probe the vibrational dynamics of a number of lyophilised and spray-dried formulations and to track the mobility of bovine serum albumin (BSA) and monoclonal antibodies (mAbs) with temperature ${ }^{18}$. Thus, for solid state systems, in line with the energy landscape model, $T_{\mathrm{g}, \beta}$, is associated with the $\beta$-process and defined as the point at which the molecules have a sufficient free energy to escape a local energy well and begin exploring different conformational environments with increasing temperature. Upon reaching $T_{\mathrm{g}, \alpha}$, associated with the $\alpha$-process as a result of further increase in temperature, the mobility of the molecules can either: (1) increase gradually, as the molecules continuously explore different conforma- tional environments, or (2) reach a plateau and become trapped in a deep energy minimum (Figure 1).

In the present study, we extended the work performed by $\mathrm{Ci}$ cerone and Soles ${ }^{19}$ and investigated whether or not the initial energy barrier that has to be overcome before local mobility can occur will be higher for spray-dried mAb formulations containing small amounts of glycerol. We also aim to answer the following questions: How does this behaviour change for higher glycerol contents? How do both the onset and the rate of these motions relate to solid state protein stability? In order to answer these questions a 52-week stability study was conducted using a selection of multi-component spray-dried mAb formulations covering a broad range of trehalose, glycerol and residual water contents that were intentionally selected to achieve a broad range of mAb stability. More specifically, a formulation containing a $5 \% \mathrm{~m} / \mathrm{m}$ glycerol/trehalose ratio was added to the study. The glycerol/trehalose ratio was chosen corresponding to and to expand on the work of Cicerone et al. ${ }^{20}$ and Bellavia et al. ${ }^{21}$, who report superior protein stability for this ratio and attributed it to an antiplasticisation effect of glycerol on the trehalose matrix.

\section{Experimental}

\subsection{Materials}

A humanised immunoglobulin G4 (IgG4) monoclonal antibody was provided by UCB Pharma (Braine l'Alleud, Belgium). D(+)trehalose dihydrate and polysorbate 20 (PS20) were obtained from Sigma-Aldrich (Steinheim, Germany). Glycerol (anhydrous), L-histidine (L-his) and L-histidine hydrochloride (LhisHCl) monohydrate were purchased from Merck (Darmstadt, Germany) and used as received.

\subsection{Spray-drying}

Feed solutions were spray-dried using a Büchi B-290 Mini Spraydryer, equipped with a $0.7 \mathrm{~mm}$ two-fluid nozzle, standard cyclone, standard collection vessel and the B-296 Dehumidifier (Büchi Labortechnik AG, Flawil, Switzerland). Feed solutions had a protein concentration of $50 \mathrm{mg} \mathrm{mL}^{-1}$. Settings were based on the methodology previously described by Batens et al. ${ }^{22}$ with the inlet air temperature set at $393 \mathrm{~K}$ (outlet temperature was monitored and ranged between $343 \mathrm{~K}$ and $353 \mathrm{~K}$ ), inlet air flow rate at $580 \mathrm{Lmin}^{-1}$, nozzle $\mathrm{N}_{2}$ flow rate at $10 \mathrm{Lmin}^{-1}$ and the solution feed rate was set at $3 \mathrm{mLmin}^{-1}$.

Powder fractions were then collected from both the collection vessel and the cyclone, both fractions were pooled together and dispensed into $2 \mathrm{~mL}$ Type I, clear, tubular glass injection vials (Schott AG, Mainz, Germany) with FluroTec ${ }^{\circledR}$ rubber injection stoppers (West Pharmaceutical Services, West Whiteland Township, PA, USA). The filled, open vials were then subjected to 2 hours of post-drying at 1 mbar and $298 \mathrm{~K}$ in an Epsilon 2$6 \mathrm{D}$ freeze-dryer (Martin Christ Gefriertrocknungsanlagen $\mathrm{GmbH}$, Osterode am Harz, Germany), followed by the application of a 1 bar nitrogen atmosphere during 10 minutes before stoppers were pushed downwards, ensuring a nitrogen atmosphere in each closed vial and capped with aluminium crimp seals (Adelphi Healthcare Packaging, West Sussex, UK). Final compositions 


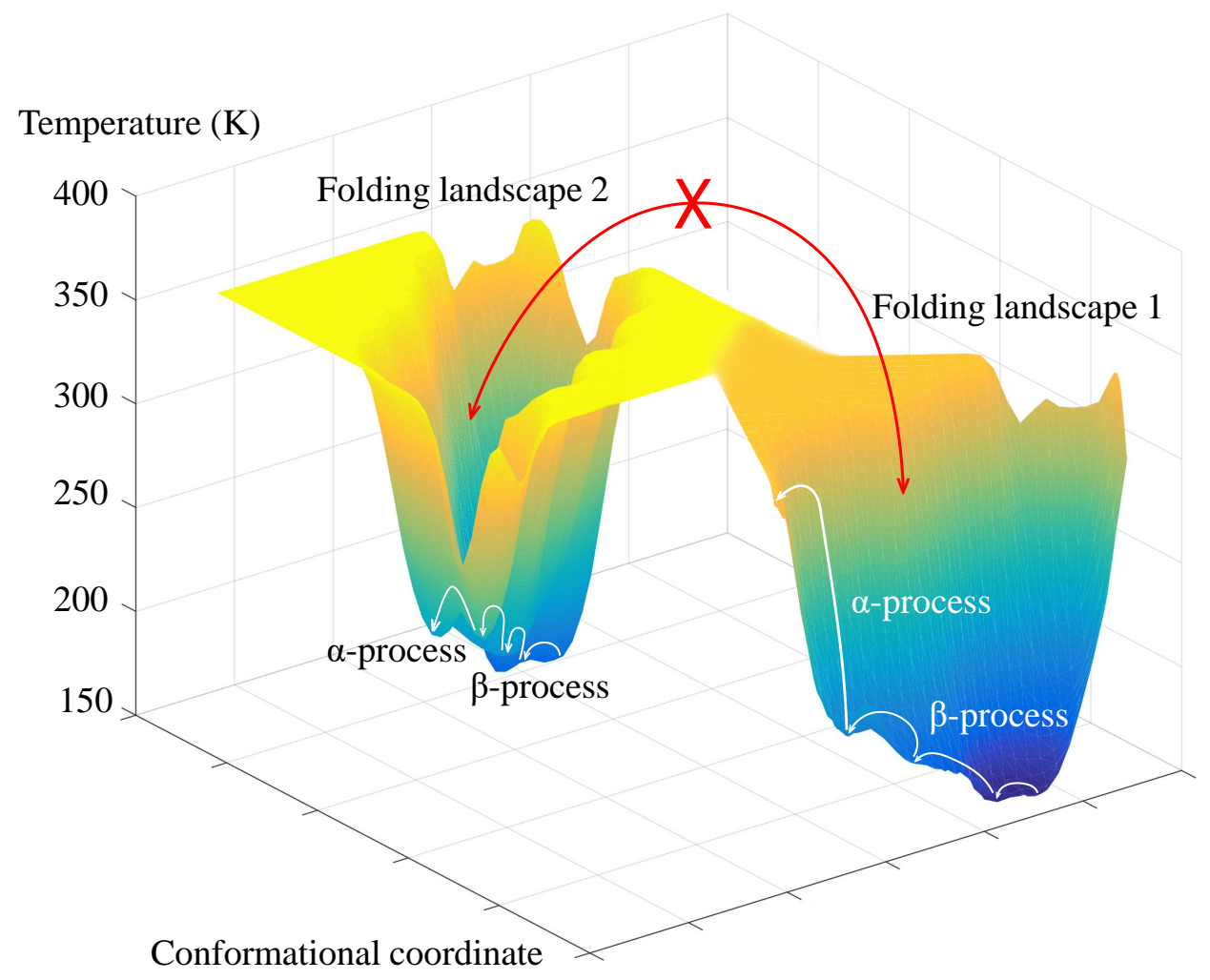

Fig. 1 Illustration of a potential energy surface showing the different minima between which a protein molecule could move in the solid state. The deepest minimum in the landscape can be linked with molecules in the most stable state. At low temperatures the protein conformation is trapped in a deep energy minimum with limited molecular mobility. With sufficient input of heat energy to the system, the $\beta$-process occurs and the protein can begin to move from one energy minimum to a different energy minimum and explore an ensemble of localised motions, e.g. small conformational changes exposing hydrophobic groups in the solid state. With additional energy input the $\alpha$-process occurs and the protein can overcome greater energy barriers and achieve larger conformational changes. As indicated on the illustration, the protein cannot move between different folding landscapes, e.g. between the native and a mis- or unfolded alternative state, in the solid matrix and generally denatures above $400 \mathrm{~K}$. It should be noted that in this illustration excipients are not included. Additionally, reconstitution of the protein in an aqueous environment will completely change the energetics of the landscape, which are traditionally depicted in a Gibbs free energy landscape. 
of the resulting powders are summarised in Table 1. Finally, mAb samples were stored either at 278,298 or $313 \mathrm{~K}$ for a 52 -week stability study.

\subsection{Physicochemical Characterisation of the Solid State}

\subsubsection{Terahertz Time-Domain Spectroscopy (THz-TDS).}

Samples were prepared for terahertz time-domain spectroscopy (THz-TDS) measurements as described by Shmool et al. ${ }^{23}$ and were acquired using the methodology introduced previously ${ }^{24}$. The changes in dynamics of the samples were analysed by investigating the change in the absorption coefficient at a frequency of $1 \mathrm{THz}$ as a function of temperature.

\subsubsection{Dynamic Mechanical Analysis (DMA).}

The TA Q800 dynamic mechanical analysis (DMA) powder clamp, i.e. the lower tray and upper cover plate accessory available for powder measurement, was used in conjunction with a TA Instruments Q800 dynamic mechanical analyser and the $35 \mathrm{~mm}$ dual cantilever clamp (TA Instruments, New Castle, DE, USA). DMA measurements were performed using argon as the air bearing gas in 'multi-frequency-strain' mode at a frequency of $1 \mathrm{~Hz}$ and an amplitude of $10 \mu \mathrm{m}$. The lower tray of the TA Q800 DMA powder clamp was filled at ambient conditions after which the cover plate was firmly pressed onto the clamp by hand. After weighing the filled clamp to allow sample mass calculation using the previously determined mass of the empty TA Q800 DMA powder clamp, the clamp was then mounted into the $35 \mathrm{~mm}$ dual cantilever clamp and this was subsequently tightened with a torque wrench using $14 \mathrm{kPa}$ of pressure. Following closing of the furnace, temperature was equilibrated at $278 \mathrm{~K}$ and kept isothermally for $15.00 \mathrm{~min}$ utes. Subsequently, temperature was equilibrated at $138 \mathrm{~K}$ and kept isothermally for 15.00 minutes before ramping the temperature at a rate of $2.00 \mathrm{Kmin}^{-1}$ until the $\alpha$-transition was observed or up to a maximum temperature of $393 \mathrm{~K}$ was reached to avoid clamp sticking due to the sample becoming liquid or degrading.

Each formulation was measured in duplicate and, even though storage modulus $\left(E^{\prime}\right)$, loss modulus $\left(E^{\prime \prime}\right)$ and $\tan \delta$ responses were all taken into consideration for data analysis, only the $\tan \delta$ or damping response was reported in this work since, by definition, it represents both moduli. In the equations $\sigma$ is defined as the applied stress, $\varepsilon$ as the applied strain and $\delta$ as the phase lag of a sample measured with DMA. The $\tan \delta$ response is a measure for the ratio of the system's potential to dissipate energy as heat and permanently deform relative to its potential to store energy and recover from deformation. Permanent deformation is associated with increased molecular mobility and the height (and amplitude) of the $\tan \delta$ response consequently serves as a measure for this as well. Additionally, the area under the $\tan \delta \beta$-transition peak is associated with the amount of energy that released during the transition and the full width at half maximum (FWHM) is a characteristic for the time distribution across the transition 25,26 .

Following data collection, $\tan \delta$ values were normalised for sample mass. The $\tan \delta \beta$-transition peaks, defined as the first transition observed in the $\tan \delta$ signal when heating from $138 \mathrm{~K}$, were then fitted using the peak analyser tool of the OriginPro
8.5.0 software package (OriginLab Corporation, Northampton, MA, USA) using the fit peaks (pro) function with baseline corrected to the minimal signal value, a smoothing window size of 10 , positive peaks identified as local maxima using 100 local points without the application of peak filtering or weighting and a 500 iteration fit control with a tolerance of $10^{-15}$. When no clear second peak, i.e. $\alpha$-transition peak, could be fitted, the right limit of the $\tan \delta \beta$-transition peak was manually set at the minimum before the $\alpha$-transition onset. Finally, the mean $(n=2)$ of the resulting peak amplitude, FWHM, area and $T_{\mathrm{g}, \beta, \mathrm{DMA}}$, defined as the temperature of the peak maximum, values were calculated for each formulation.

\subsection{Powder Reconstitution}

Spray-dried powders were reconstituted at $100 \mathrm{mg} \mathrm{mL}^{-1}$ by injecting a calculated volume of ultrapure water through the closed vials' septa using a syringe and 18 gauge needle. Following water addition, vials were swirled gently to evenly distribute the solvent. Aside from the initial, gentle swirl, samples were not agitated. After reconstitution, the mAb concentration of the solutions was verified using UV absorbance at $280 \mathrm{~nm}$ using an extinction coefficient of $1.33 \mathrm{mLmg}^{-1} \mathrm{~cm}^{-1}$.

\subsection{2-Week Stability Study}

In order to evaluate the solid state (storage) stability of the spray-dried mAb formulations, a 52-week stability study was performed. The closed vials of the spray-dried $\mathrm{mAb}$ formulations that were kept sealed inside a 1 bar nitrogen atmosphere were stored at 278, 298 and $313 \mathrm{~K}$. At each time point (see Electronic Supplementary Information (ESI) ${ }^{\dagger}$ ), a number of closed vials were collected from their dedicated storage condition and placed at $278 \mathrm{~K}$ before analysis. The spray-dried $\mathrm{mAb}$ formulations were subsequently subjected to solid state characterisation or reconstituted to $100 \mathrm{mg} \mathrm{mL}^{-1}$ for aggregation-based stability assessment. Solid state characterisation at each time point consisted of residual water content determination (KF), verifying sample amorphicity (XRPD and DSC), thermal (DSC) and spectral analysis (FTIR). Aggregation-based stability assessment of the reconstituted samples consisted of determining the high molecular weight species (HMWS) and large aggregate content, using SEC and DLS, respectively, and measuring the optical density at incident wavelength of $600 \mathrm{~nm}$ (OD600) as a measure for sample turbidity.

Following data collection for all time points, HMWS, large aggregates and turbidity were fitted linearly as a function of time using the LINEST function included in the Microsoft ${ }^{\circledR}$ Excel $^{\circledR}$ 2016 software package version 16 (Microsoft Corporation, Redmond, WA, USA) in order to obtain gradient values (d HMWS $/ \mathrm{d} t$ ) for each formulation and storage condition. The obtained gradient values were consequently used as quantitative parameters to measure solid state mAb instability.

\subsubsection{Size-Exclusion Chromatography (SEC).}

Size-exclusion chromatography (SEC) was used to quantify multimeric/high molecular weight species (HMWS) (i.e. dimers, trimers, tetramers,...) in the reconstituted samples. HMWS were 
Table 1 Composition of the spray-dried mAb formulations

\begin{tabular}{lccccccc}
\hline & $\begin{array}{c}\mathrm{mAb} \\
\% \mathrm{~m} / \mathrm{m}\end{array}$ & $\begin{array}{c}\text { Tre } \\
\% \mathrm{~m} / \mathrm{m}\end{array}$ & $\begin{array}{c}\text { L-Gly } \\
\% \mathrm{~m} / \mathrm{m}\end{array}$ & $\begin{array}{c}\text { PS20 } \\
\% \mathrm{~m} / \mathrm{m}\end{array}$ & $\begin{array}{c}\text { L-His } \\
\% \mathrm{~m} / \mathrm{m}\end{array}$ & $\begin{array}{c}\text { L-His-HCl } \\
\% \mathrm{~m} / \mathrm{m}\end{array}$ & $\begin{array}{c}\text { Water } \\
\% \mathrm{~m} / \mathrm{m} \\
\pm \text { SD }\end{array}$ \\
\hline F1 & 63.13 & 31.57 & 0 & 0.25 & 0.66 & 2.81 & 1.57 \\
F2 & 63.30 & 15.83 & 15.83 & 0.25 & 0.67 & 2.82 & 1.31 \\
F3 & 63.48 & 0 & 31.74 & 0.25 & 0.67 & 2.82 & 1.04 \\
F4 & 62.22 & 31.11 & 1.56 & 0.25 & 0.65 & 2.77 & 1.45 \\
\hline
\end{tabular}

defined as the percentage of the total area eluting before the monomer peak and are a measure for the loss of monomeric mAb. Analysis was performed using an Infinity 1260 high performance liquid chromatography (HPLC) system (Agilent Technologies, Waldbronn, Germany) equipped with a TSK-GEL G3000SW ${ }_{X L}$ column (5 $\mu \mathrm{m}, 300 \mathrm{~mm}$ x $7.8 \mathrm{~mm}$ ) (Tosoh Biosciences, Germany) and UV-detector (set at $280 \mathrm{~nm}$ ). A filtered $0.2 \mathrm{M}$ sodium phosphate ( $\mathrm{pH}$ 7.0) solution, was used as the mobile phase. Samples were diluted to $5 \mathrm{mg} \mathrm{mL}^{-1}$ using mobile phase and $50 \mu \mathrm{l}$ were injected onto the column. The process was run isocratically at ambient temperature, for 15 minutes with a flow rate of $1 \mathrm{mLmin}^{-1}$ and two injections were performed for each sample. Peaks were integrated with Empower (version 7.30.00.00) and the ApexTrack ${ }^{\circledR}$ algorithm (Waters Corporation, Milford, MA, USA).

\section{Results}

Overall, a relative increase in the high molecular weight species (HMWS) content over time was observed for all formulations. The aggregation rate ( $\mathrm{dHMWS} / \mathrm{d} t$ ) was used as the indicator for the stability of the formulation, with high values indicating poor stability. The increase in $\mathrm{d} H \mathrm{HWS} / \mathrm{d} t$ was most pronounced for formulation F3, i.e. the formulation with the highest glycerol content that did not contain any trehalose.

\subsection{Terahertz Time-Domain Spectroscopy (THz-TDS)}

For all of the formulations, the change in absorption coefficient $(\alpha)$ with temperature (T) was observed to take place over three distinct thermal regions that were separated by two transition temperatures, $T_{\mathrm{g}, \beta, \mathrm{THz}}$ and $T_{\mathrm{g}, \alpha, \mathrm{THz}}$ (Table 2). The gradient of each distinct linear region is denoted as $\mathrm{d} \alpha_{1} / \mathrm{d} T, \mathrm{~d} \alpha_{2} / \mathrm{d} T$ and $\mathrm{d} \alpha_{3} / \mathrm{d} T$ representing regions 1,2 , and 3 , respectively, with region 1 being the lowest and region 3 being the highest temperature region.. All characteristic parameters of the linear fit based on terahertz analysis were clearly influenced by the excipient composition of the spray-dried mAb formulations (Figure 2).

\subsection{Dynamic Mechanical Analysis (DMA)}

For all formulations a set of characteristic parameters of the $\tan \delta$ $\beta$-transition peak was determined (Table 3 ). The amplitude and area (Figure 3 ) of the fitted mean $\tan \delta \beta$-transition peak showed a clear, positive correlation with the glycerol content of the spraydried mAb.

\section{Discussion}

$T_{g, \beta}$ represents the thermal energy required to overcome the lowest local energy barrier on the PES. The depth of this minimum can be characterised by the quantity of energy (heat) required to be added to the system before additional states can be explored. Given that the energy requirements to overcome the minimum at $T_{\mathrm{g}, \beta}$ were met for all formulations stored at $313 \mathrm{~K}$, it is important to consider the observed differences in solid state stability (d HMWS $/ \mathrm{d} t$ ) over time. Given that $\mathrm{d} \alpha_{2} / \mathrm{d} T$ represents a gradient of the amplitude of excess (non-harmonic) motion and the shape of the surface of the energy landscape between minima, we emphasise that not only the depth and presence of energy minima, but also the roughness of the PES dictates the stability of proteins in the solid state. A rougher surface, displaying a large number of relatively shallow energy minima, present across the entire surface, as well as rough surfaces within the deeper energy wells, will cause more hindrance for the molecules to explore alternative conformational states (which are less kinetically accessible) ${ }^{8,27}$. This impact on the process kinetics can be compared to the increased energy required to push a cart down a rough cobblestone surface versus down a smooth asphalt surface. Specifically, the changes in protein motions associated with the $\beta$ process would occur on a relatively fast time-scale due to the lowlying energy barriers between different energy wells. The high energy barriers associated with the $\alpha$-relaxation result in slower kinetics observed for this process. Therefore, we propose that not only the onset and depth of the energy minima characterised by $T_{\mathrm{g}, \beta}$, but also the roughness of the PES, i.e. the gradient by which the amplitude of excess motion increases with increasing temperature, characterised by $\mathrm{d} \alpha_{2} / \mathrm{d} T$, govern protein stability in the solid state.

Large conformational changes or aggregate formation above the level of dimers are highly unlikely in the solid state, as illustrated by the work of Koshari et al. ${ }^{28}$. However, as outlined by Cicerone and Douglas ${ }^{5}$, small conformational changes exposing hydrophobic groups could increase the propensity for aggregation upon reconstitution in a hydrophilic medium ${ }^{3}$. It is reasonable to assume that these more hydrophobic, aggregation-prone conformations will be energetically less unfavourable in the solid state as water is removed during drying. Upon reconstitution, the most favourable state will depend on the conformational state of the protein, e.g. for a structure with more exposed hydrophobic residues or a partially unfolded structure, the aggregated form will be the most favourable because of the reduction of hydrophobic groups exposed to the hydrophilic solvent. Due to the inherently lower mobility requirements for the small conformational 
Table 2 Based on the terahertz analysis of the spray-dried mAb formulations, three distinct linear regions could be identified for the absorption coefficient $\alpha$ as a function of temperature (T). The gradient of each distinct linear region is denoted as $\mathrm{d} \alpha_{1} / \mathrm{d} T, \mathrm{~d} \alpha_{2} / \mathrm{d} T$ and $\mathrm{d} \alpha_{3} / \mathrm{d} T$ representing regions 1,2 , and 3 , respectively. The inflection points between regions 1 and 2 and regions 2 and 3 are labelled as $T_{\mathrm{g}, \beta, \mathrm{THz}}$ and $T_{\mathrm{g}, \alpha, \mathrm{THz}}$, respectively.

\begin{tabular}{|c|c|c|c|c|c|c|c|c|}
\hline & \multicolumn{2}{|c|}{$\begin{array}{c}\mathrm{d} \alpha_{1} / \mathrm{d} T \\
\mathrm{~cm}^{-1} \mathrm{~K}^{-1}\end{array}$} & \multicolumn{2}{|c|}{$\begin{array}{c}\mathrm{d} \alpha_{2} / \mathrm{d} T \\
\mathrm{~cm}^{-1} \mathrm{~K}^{-1}\end{array}$} & \multicolumn{2}{|c|}{$\begin{array}{c}\mathrm{d} \alpha_{3} / \mathrm{d} T \\
\mathrm{~cm}^{-1} \mathrm{~K}^{-1}\end{array}$} & \multirow{2}{*}{$\begin{array}{c}T_{\mathrm{g}, \beta, \mathrm{THz}} \\
\mathrm{K} \\
n=3\end{array}$} & \multirow{2}{*}{$\begin{array}{c}T_{\mathrm{g}, \alpha, \mathrm{THz}} \\
\mathrm{K} \\
n=3\end{array}$} \\
\hline & $n=3$ & $\pm \mathrm{SD}$ & $n=3$ & $\pm \mathrm{SD}$ & $n=3$ & $\pm \mathrm{SD}$ & & \\
\hline$\overline{\mathrm{F} 1}$ & 0.002 & 0.0032 & 0.043 & 0.0009 & 0.08 & 0.0035 & 178 & 268 \\
\hline F2 & 0.016 & 0.00097 & 0.054 & 0.0010 & 0.10 & 0.0031 & 209 & 343 \\
\hline F3 & 0.009 & 0.0032 & 0.120 & 0.0048 & 0.19 & 0.0071 & 222 & 334 \\
\hline F4 & 0.021 & 0.0036 & 0.055 & 0.0024 & 0.04 & 0.0010 & 190 & 344 \\
\hline
\end{tabular}

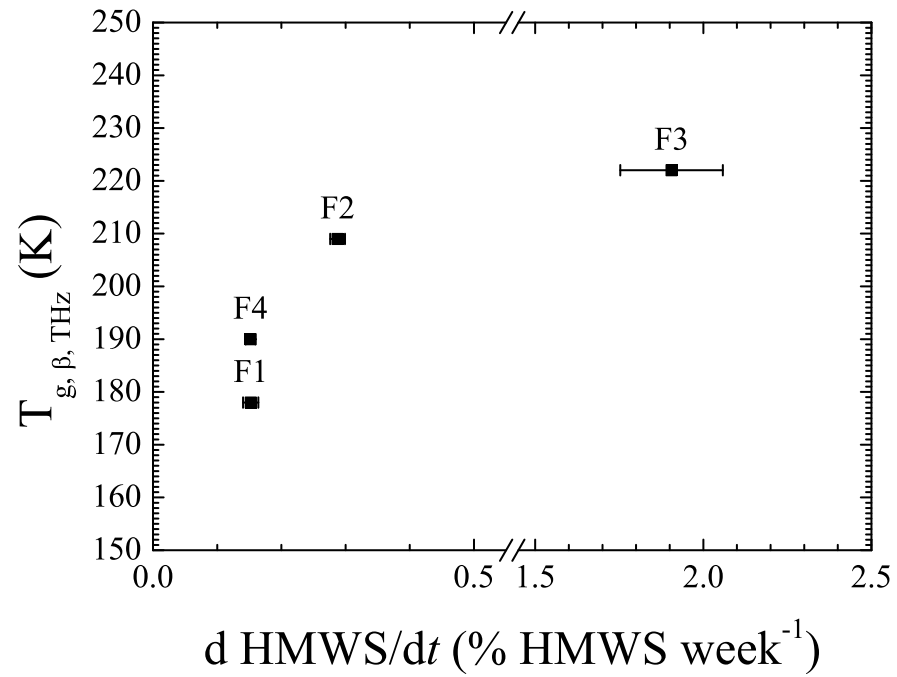

(a)

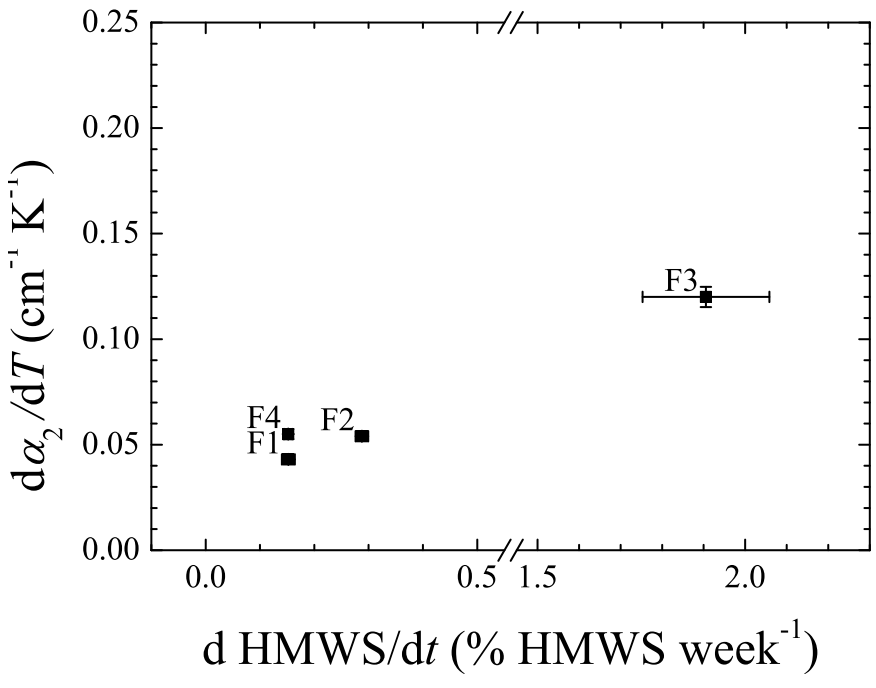

(b)

Fig. 2 (a) $T_{\mathrm{g}, \beta, \mathrm{THz}}$ and (b) $\mathrm{d} \alpha_{2} / \mathrm{d} T$ as a function of $\mathrm{d} \mathrm{HMWS} / \mathrm{d} t$ for samples stored at $313 \mathrm{~K}$. Horizontal error bars depict the standard error of the slope as a measure for the precision of the regression analysis. Vertical error bars depict the standard deviation for $n=3$ samples.

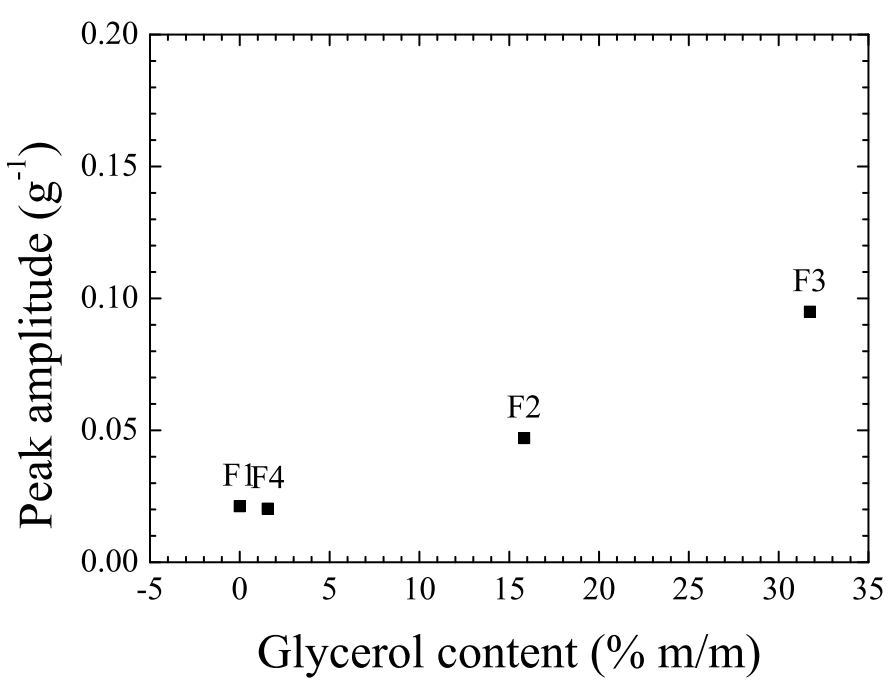

(a)

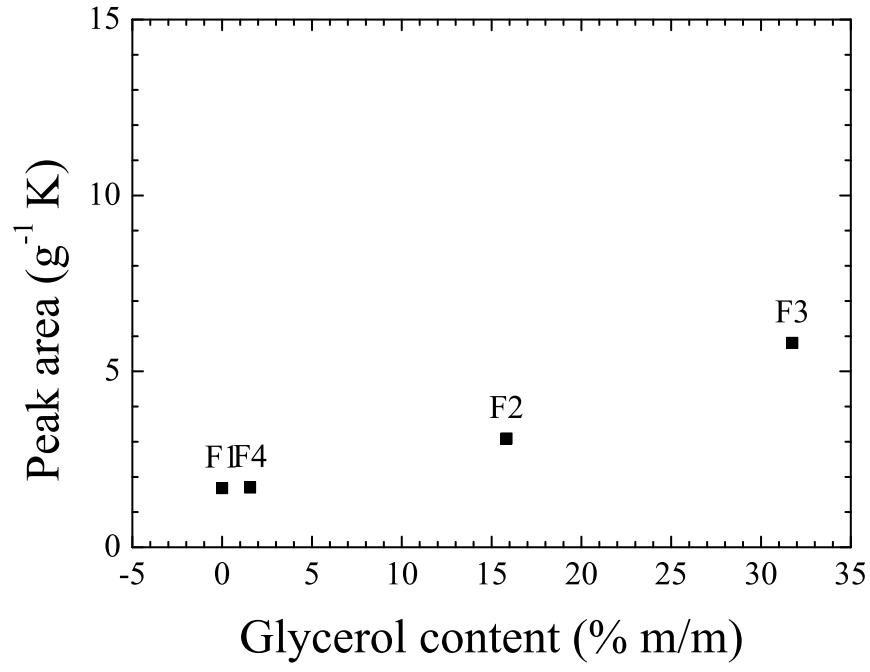

(b)

Fig. 3 (a) The amplitude and (b) area of the fitted mean $(n=2) \tan \delta \beta$-transition peak based on DMA as a function of the glycerol content of formulations $F 1, F 2, F 3$ and F4, respectively. Values were determined on $t_{0}$ samples stored at $278 \mathrm{~K}$. 
Table 3 Characteristic parameters of the fitted mean $(n=2) \tan \delta \beta$ transition peak based on DMA of spray-dried mAb formulations.

\begin{tabular}{lcccc}
\hline & $\begin{array}{c}\text { Peak amplitude } \\
\mathrm{g}^{-1}\end{array}$ & $\begin{array}{c}\text { FWHM } \\
\mathrm{K}\end{array}$ & $\begin{array}{c}\text { Peak area } \\
\mathrm{g}^{-1} \mathrm{~K}\end{array}$ & $\begin{array}{c}T_{\mathrm{g}, \beta, \text { DMA }} \\
\mathrm{K}\end{array}$ \\
\hline F1 & 0.0212 & 74.47 & 1.68 & 227.17 \\
F2 & 0.0471 & 62.08 & 3.09 & 224.20 \\
F3 & 0.0950 & 57.45 & 5.81 & 232.95 \\
F4 & 0.0203 & 78.53 & 1.70 & 226.29 \\
\hline
\end{tabular}

changes, these are possible at $T_{\mathrm{g}, \beta}$. Therefore transitioning between the initial and more aggregation-prone conformations is possible in the dehydrated matrix and during storage due to the limited mobility requirements associated with small conformational changes, but to which degree will also depend on the local environment of the protein, e.g. the localised presence or absence of residual water. Notably, it has been reported that, while ultimately driven by thermodynamics, protein aggregation is generally strongly kinetically controlled ${ }^{29-31}$. In this context, the native state of the protein of monomeric proteins was suggested to be metastable with respect to the aggregated state ${ }^{31}$. It should be mentioned that the referenced research focussed on the amyloid state, a specific form of aggregates characterised by a fibrillar morphology arising from certain proteins which has not been reported for mAbs. However, the principles put forward can easily be expanded to other forms of aggregates as well. In the PES, a low energy state is represented as a deep minimum. From an energy landscape perspective, with heating, at $T_{\mathrm{g}, \beta}$ parts of the protein can mobilise between energy minima from one low-energy conformation to another. The presence of these higher energy barriers is in line with the higher $T_{\mathrm{g}, \beta}$ and $T_{\mathrm{g}, \alpha}$ values measured for formulation F3 and, to a lesser extent, F2 (Figure 2a), which contained a higher amount of HMWS following spray-drying and reconstitution.

The extent of mobility of the system is largely determined by the role of the excipients and their interactions with the protein. However, as is apparent from Figure $2 \mathrm{~b}$ and the ESI, predictions of solid state stability of spray-dried protein formulations should not be based on the values of $T_{\mathrm{g}, \beta}$ and $T_{\mathrm{g}, \alpha}$ alone. Therefore, we propose that additional parameters defining the extent of mobility or gradient of the amplitude of excess motion of the system should be considered. To highlight, from THz-TDS data, we suggest $\mathrm{d} \alpha_{2} / \mathrm{d} T$ should be further examined, while for DMA experiments additional research should be performed to assess the exact physical meaning of the area under the $\tan \delta \beta$-transition peak and its relation to the extent of mobility or gradient of the amplitude of excess motion. Figure $2 \mathrm{~b}$ shows an increase in the value of $\mathrm{d} \alpha_{2} / \mathrm{d} T$ for F2 and F3, indicating that glycerol increases the relative molecular mobility and flexibility of the system ${ }^{5,19}$. Furthermore, the THz-TDS spectra show that above $T_{\mathrm{g}, \alpha}$, Region 3 has the lowest gradient value for F4 and F1 followed by F2, and F3 has the highest value for $\mathrm{d} \alpha_{3} / \mathrm{d} T$. This suggests that with increasing temperature, hydrogen bonds between the mAb and a stiff matrix, i.e. restricted mobility, could reduce the conformational mobility of the mAb and increase its solid state stability at elevated temperatures. This is corroborated by a higher glycerol content resulting in an increased amplitude of the $\tan \delta$ $\beta$-transition peak (Figure 3a), which serves as a measure for increased molecular mobility. Additionally, the amount of energy released during the $\beta$-transition increased with higher glycerol content, as indicated by the increase in area under the $\tan \delta \beta$ transition peak (Figure $3 \mathrm{~b}$ ), an additional characteristic of the roughness of the PES ${ }^{25,26}$. These observations are consistent with previous work which shows that low mobility is related to high formulation stability, and here F1 and F4, which exhibit the lowest mobility above $T_{\mathrm{g}, \alpha}$, as indicated by the value of the gradient, also show the highest stability following prolonged storage.

\section{Conclusions}

This work raises the question: What is required for a protein to resist conformational changes in the solid state? The first is that the protein exists in a low energy conformation in the solid state. In the second case, the protein may exist in a higher energy conformation after water removal, but is stabilised by the interactions formed between the protein and excipients, i.e. the achieved stability is a consequence of kinetic factors rather than thermodynamic ones. It can be proposed that this is the case for proteins in energetically favourable, aggregation-prone conformations, or a system which is highly stable, due to strong protein and excipient interactions. These are the systems which exhibit high values for $T_{\mathrm{g}, \beta}$ and resist conformational changes induced by heat. Therefore, if protein molecules are able to reach a minimum above $T_{g, \alpha}$, they exist in a relatively stable conformation, compared to a system in which the protein molecules do not reach a minimum above $T_{g, \alpha}$. The presented data offer a more comprehensive explanation for the stabilising properties observed for small flexible molecules. When examining a glycerol-containing solid state protein formulation, it is critical to consider the mutual interplay between thermodynamics and kinetics of the system: the transition temperatures $\left(T_{g}\right)$ and parameters characterising the gradient of the amplitude of excess motion. Additionally, we suggest further work should be completed, extending the presented hypothesis on the fundamental nature of the processes discussed in this work and how these link to protein stability in the solid state for amorphous and co-amorphous formulations.

\section{Acknowledgements}

The authors would like to thank UCB Pharma for providing the equipment, materials and funding to make this study possible.

T.A.S. and J.A.Z. acknowledge funding from AstraZeneca UK Limited/MedImmune Limited and the UK Engineering and Physical Sciences Research Council (EP/N022769/1). T.A.S. would like to thank the AJA-Karten Trust and the AIA-Kenneth Lindsay Trust for their financial support.

\section{Notes and references}

1 B. C. Hancock and G. Zografi, Journal of Pharmaceutical Sciences, 1997, 86, 1-12.

2 S. Yoshioka and Y. Aso, Journal of Pharmaceutical Sciences, 2007, 96, 960-981.

3 M. T. Cicerone, M. J. Pikal and K. K. Qian, Advanced Drug Delivery Reviews, 2015, 93, 14-24. 
4 T. E. Dirama, J. E. Curtis, G. A. Carri and A. P. Sokolov, The Journal of Chemical Physics, 2006, 124, 034901.

5 M. T. Cicerone and J. F. Douglas, Soft Matter, 2012, 8, 29832991.

6 S. Magazù, F. Migliardo and A. Benedetto, The Journal of Physical Chemistry B, 2011, 115, 7736-7743.

7 P. W. Fenimore, H. Frauenfelder, S. Magazù, B. H. McMahon, F. Mezei, F. Migliardo, R. D. Young and I. Stroe, Chemical Physics, 2013, 424, 2-6.

8 B. Varga, F. Migliardo, E. Takacs, B. Vertessy, S. Magazù and M. T. F. Telling, Journal of Biological Physics, 2010, 36, 207220.

9 K. L. Ngai, Philosophical Magazine, 2004, 84, 1341-1353.

10 S. Capaccioli, K. L. Ngai, S. Ancherbak and A. Paciaroni, The Journal of Physical Chemistry B, 2012, 116, 1745-1757.

11 K. L. Ngai, S. Capaccioli and A. Paciaroni, The Journal of Chemical Physics, 2013, 138, 235102.

12 A. Ottochian, C. D. Michele and D. Leporini, The Journal of Chemical Physics, 2009, 131, 224517.

13 J. C. Dyre, Physical Review E, 2013, 88, 042139.

14 J.-H. Hung, T. K. Patra, V. Meenakshisundaram, J. H. Mangalara and D. S. Simmons, Soft Matter, 2019, 15, 1223-1242.

15 M. T. Ruggiero, M. Krynski, E. O. Kissi, J. Sibik, D. Markl, N. Y. Tan, D. Arslanov, W. van der Zande, B. Redlich, T. M. Korter, H. Grohganz, K. Lobmann, T. Rades, S. R. Elliott and J. A. Zeitler, Physical Chemistry Chemical Physics, 2017, 19, 30039-30047.

16 G. P. Johari, The Journal of Physical Chemistry B, 2019, 123, 3010-3023.

17 M. T. Cicerone and M. Tyagi, The Journal of Chemical Physics, 2017, 146, 054502.

18 T. A. Shmool and J. A. Zeitler, Polymer Chemistry, 2019, 10, 351-361.

19 M. T. Cicerone and C. L. Soles, Biophysical Journal, 2004, 86, 3836-45.

20 M. T. Cicerone, A. Tellington, L. Trost and A. Sokolov, Bioprocess International, 2003, 1, 36-47.

21 G. Bellavia, L. Paccou, Y. Guinet and A. Hédoux, The Journal of Physical Chemistry B, 2014, 118, 8928-8934.

22 M. Batens, L. Dewaele, C. Clasen, J. Massant and G. Van den Mooter, Electrospraying Fully Aqueous Protein Solutions: A Feasibility Assessment, 2018.

23 T. A. Shmool, P. J. Woodhams, M. Leutzsch, A. D. Stephens, M. U. Gaimann, M. D. Mantle, G. S. Kaminski Schierle, C. F. van der Walle and J. A. Zeitler, International Journal of Pharmaceutics: X, 2019, 1, 100022.

24 T. A. Shmool, M. Batens, J. Massant, G. Van den Mooter and J. A. Zeitler, European Journal of Pharmaceutics and Biophar- maceutics, 2019, 144, 244-251.

25 K. Menard, Dynamic Mechanical Analysis: A Practical Introduction, CRC Press LLC, Boca Raton, FL, US, 1st edn., 1999.

26 K. P. Menard and N. R. Menard, in Encyclopedia of Polymer Science and Technology, American Cancer Society, Atlanta, GA, US, 2015, pp. 1-33.

27 M. C. Prentiss, D. J. Wales and P. G. Wolynes, PLoS Computational Biology, 2010, 6, e1000835.

28 S. H. S. Koshari, P. K. Nayak, S. Burra, I. E. Zarraga, K. Rajagopal, Y. Liu, N. J. Wagner and A. M. Lenhoff, Molecular Pharmaceutics, 2019, 16, 173-183.

29 D. Thirumalai and G. Reddy, Nature Chemistry, 2011, 3, 910 911.

30 A. J. Baldwin, T. P. J. Knowles, G. G. Tartaglia, A. W. Fitzpatrick, G. L. Devlin, S. L. Shammas, C. A. Waudby, M. F. Mossuto, S. Meehan, S. L. Gras, J. Christodoulou, S. J. Anthony-Cahill, P. D. Barker, M. Vendruscolo and C. M. Dobson, Journal of the American Chemical Society, 2011, 133, 14160-14163.

31 P. Ricchiuto, A. V. Brukhno and S. Auer, The Journal of Physical Chemistry B, 2012, 116, 5384-5390.

\section{Contents of the $\mathrm{ESI}^{\dagger}$}

Supplementary Methods.

Supplementary Results.

Fig. S1. FTIR spectra.

Fig. S2. SEC, DLS and OD600 data as a function of time.

Fig. S3. d HMWS/dt as a function of the glycerol content.

Fig. S4. $T_{\mathrm{g}, \alpha, \mathrm{DSC}}$ as a function of the glycerol content and $\mathrm{d}$ HMWS/d $t$.

Fig. S5. Mean terahertz absorption coefficient as a function of temperature at $1 \mathrm{THz}$.

Fig. S6. Characteristic THz-TDS parameters as a function of the glycerol content and d HMWS/d $t$.

Fig. S7. $\tan \delta$ responses collected using DMA.

Fig. S8. Characteristic DMA parameters as a function of the glycerol content and d HMWS/d .

Table S1. d HMWS/d $t$ values of samples stored at 278, 298 and $313 \mathrm{~K}$.

Table S2. Mean $T_{\mathrm{g}, \alpha, \mathrm{DSC}}$ values. 